\title{
Optical Trap
}

National Cancer Institute

\section{Source}

National Cancer Institute. Optical Trap. NCI Thesaurus. Code C62319.

A method utilized for the non-mechanical manipulation of nanoparticles. Small transparent objects can be isolated or fixed using focused laser light. The optical trap is based on the concept of photon momentum transfer and has many applications. 